\title{
纳米粒子超晶格的非经典晶化路径
}

\author{
齐利民 \\ 北京大学化学与分子工程学院, 北京分子科学国家研究中心, 北京 100871 \\ E-mail: liminqi@pku.edu.cn
}

\section{Nonclassical crystallization pathways of nanoparticle superlattices}

\section{Limin Qi}

Beijing National Laboratory for Molecular Sciences, College of Chemistry and Molecular Engineering, Peking University, Beijing 100871, China E-mail: liminqi@pku.edu.cn

doi: 10.1360/TB-2019-0789

有关晶化路径的研究一直在材料学中占据着重要地 位, 它广泛影响着晶体材料的性质和功能 ${ }^{[1]}$. 在传统的晶 化路径(classical crystallization pathway)中, 单体会在局域 聚集并转化为另外一个相, 伴随着密度和结构有序度的增 加. 近期的实验和理论指出, 很多晶化过程并不像传统的 晶化路径那么简单, 而是经过了两个或者多个步骤才转化 为最终的结晶态. 如何从实验上观测和理解这些过渡态的 结构成为了非经典晶化路径 (nonclassical crystallization pathway)的重要问题. Nature Materials于2019年10月在线 发表了一篇实验上直接观测纳米颗粒的非经典晶化路径 的文章, 由美国伊利诺伊大学厄巴纳香槟分校的陈倩课题 组和美国西北大学的Luijten课题组 ${ }^{[2]}$ 合作完成. 他们利用 液相透射电子显微镜(liquid-phase TEM) 技术结合蒙特卡 洛模拟方法揭示了三角片状纳米颗粒在溶液中自组装形
成超晶格或晶体结构的全过程(图1).

相变过程是指材料从一个相向另外一个相的转变, 而晶化则描述具有周期结构的晶体从无序的气态或者液 态中产生的过程. 通过理解结晶路径和晶体的生长过程, 晶体的形状、结构以及均一度都可以被精确控制，该理论 也被广泛地运用于颗粒的合成和生物医药等领域. 值得一 提的是, 从2000年开始利用微米尺度颗粒体系在光学显微 镜下研究材料相变过程为理解微观动力学提供了很多丰 富的观测结果 ${ }^{[3]}$, 而能否将同一思路运用到纳米尺度的颗 粒也成为了材料学的一个重要研究方向.

传统的晶化理论是基于体系吉布斯自由能的演化来 预测晶化路径. 晶化过程中, 固相的化学势最低, 但是在 固相的形成过程中, 会产生新的界面而使体系的自由能增 加. 近期理论和实验观测发现, 晶化初期过渡态的存在可

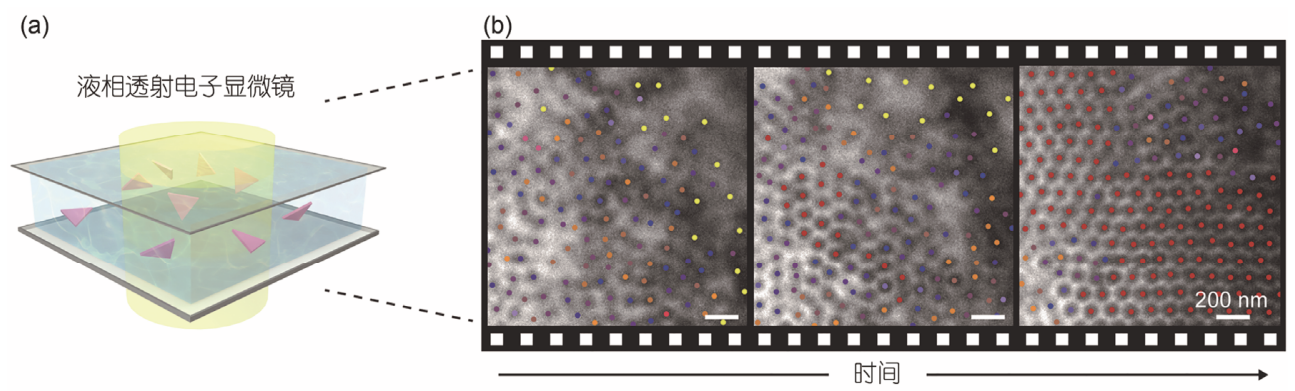

图 1 (网络版彩色)液相透射电子显微镜中观测的纳米颗粒非经典晶化路径 ${ }^{[2]}$. (a) 利用液相透射电子显微镜研究纳米颗粒在溶液中的组装动 力学的示意图; (b) 液相透射电子显微镜下观测到的非经典晶化路径, 其中圆点表示纳米颗粒的中心位置, 并用颜色表示局域的结构序参量 (有序度按黄-蓝-红的顺序增加)

Figure 1 (Color online) Nonclassical crystallization pathways of nanoparticle superlattices captured by liquid-phase TEM ${ }^{[2]}$. (a) Schematic illustration of in situ observation of the self-assembly of gold nanoprisms in solution using liquid-phase TEM. (b) Time-lapse TEM images showing the real-time crystallization process, with "gas" columns colored yellow, "liquid" columns colored blue, and "solid" columns colored red 
以降低体系的能垒, 而这对理解晶体的结构和产率有很大 帮助 ${ }^{[4]}$. 然而, 初期颗粒团簇 (particle cluster) 的尺寸在纳 米尺度, 这使得在液体中直接观测颗粒团簇的涨落和演化 很困难. 由于传统的光学方法无法分辨<200 $\mathrm{nm}$ 的颗粒, 原子力显微镜和液相电子显微镜的高空间分辨率吸引了 很多研究者的注意 ${ }^{[5,6]}$. 在之前的液相电子显微镜工作中, 由于存在基底对于颗粒动力学行为的显著影响 ${ }^{[7]}$, 无法直 接观测到热力学稳定的纳米粒子超晶格.

陈倩课题组和Luijten课题组 ${ }^{[2]}$ 对纳米尺度晶化动力学 的实验研究工作, 进一步推进了科学界基于液相透射电子 显微镜的材料研究. 首先, 实现了热力学稳定的纳米粒子 超晶格的实验观测, 并利用蒙特卡洛模拟进行了验证和热 力学解释; 其次, 基于纳米颗粒单体尺度的分辨率, 过渡 态的结构被精确地量化; 再次, 通过实验上对晶化过程的 可重复的多次观测, 发现了颗粒团簇随时间的演变过程符 合两步晶化(two-step crystallization)的模型. 值得一提的 是, 这一晶化路径在蛋白质的结晶过程模拟中被首次提 出 $^{\left[{ }^{8]}\right.}$, 之后的理论和实验工作指出该路径相较于经典晶化 路径可以降低结晶过程的能垒, 因此结晶体系在晶化过程 中会倾向于选择该能垒较低的路径 ${ }^{[9,10]}$.

这项液相透射电子显微镜观测工作为深人理解纳米
颗粒晶化过程提供了新的研究思路和方法. 由于纳米颗粒 和溶液分子的尺寸很接近, 纳米粒子之间的相互作用非常 复杂, 除了范德华作用和长程静电作用外, 还需要考虑颗 粒表面高分子配体之间的相互作用以及流体力学作用等, 这使得理论上直接模拟纳米粒子的复杂集体行为十分困 难 ${ }^{[11]}$. 而利用实验观测和统计物理学方法, 可以定量测得 纳米颗粒之间的相互作用和团簇表面张力等物理量, 从而 为理论和模拟提供重要的实验参考. 此外, 根据StokesEinstein方程, 纳米颗粒在溶液中的布朗运动较之微米颗 粒大几个量级, 这也使得直接观测各种长时间尺度行为 (如玻璃化转变)成为了可能.

需要指出的是, 液相透射电子显微镜可以广泛地运 用到不同的材料和系统中. 与超分辨光学显微镜(superresolution optical microscopy)不同的是，电子显微镜是基 于材料本身和环境的电子密度不同来产生衬度, 这意味着 该方法可以推广至有机微粒(如蛋白质)体系的动力学研究. 除此之外, 液体环境的组分、温度等都可以利用外场进行 调控, 这也意味着在液体环境中可以进一步研究纳米材料 的结构和功能对于环境变化的响应. 可以期待, 该技术未 来在材料学、物理学和生物学研究中将产生出更多有趣而 重要的结果.

\section{参考文献}

1 De Yoreo J J, Gilbert P U P A, Sommerdijk N A J M, et al. Crystallization by particle attachment in synthetic, biogenic, and geologic environments. Science, 2015, 349: aaa6760

2 Ou Z, Wang Z, Luo B, et al. Kinetic pathways of crystallization at the nanoscale. Nat Mater, 2019, doi: 10.1038/s41563-019-0514-1

3 Li B, Zhou D, Han Y. Assembly and phase transitions of colloidal crystals. Nat Rev Mater, 2016, 1: 15011

4 Erdemir D, Lee A Y, Myerson A S. Nucleation of crystals from solution: Classical and two-step models. Acc Chem Res, 2009, 42: 621-629

5 Chung S, Shin S H, Bertozzi C R, et al. Self-catalyzed growth of S layers via an amorphous-to-crystalline transition limited by folding kinetics. Proc Natl Acad Sci USA, 2010, 107: 16536-16541

6 De Yoreo J J, Sommerdijk N A J M. Investigating materials formation with liquid-phase and cryogenic TEM. Nat Rev Mater, 2016, 1: 16035

7 Kim J, Ou Z, Jones M R, et al. Imaging the polymerization of multivalent nanoparticles in solution. Nat Commun, 2017, 8: 761

8 Ten Wolde P R, Frenkel D. Enhancement of protein crystal nucleation by critical density fluctuations. Science, 1997, 277: 1975-1978

9 Lutsko J F, Nicolis G. Theoretical evidence for a dense fluid precursor to crystallization. Phys Rev Lett, 2006, 96: 046102

10 Savage J R, Dinsmore A D. Experimental evidence for two-step nucleation in colloidal crystallization. Phys Rev Lett, 2009, 102: 198302

11 Batista C A S, Larson R G, Kotov N A. Nonadditivity of nanoparticle interactions. Science, 2015, 350: 1242477 